Proceedings

\title{
Determination of the Theoretical Spectrum of Gamma Emission Generated by DGNAA in Samples of Ashes of Thermal Power Plants ${ }^{\dagger}$
}

\author{
German Marcos-Robredo ${ }^{1, *}$, M. Pilar Castro-García ${ }^{1}$, Miguel Ángel Rey-Ronco ${ }^{1}$ and \\ Teresa Alonso-Sánchez ${ }^{2}$ \\ 1 Department of Energy, University of Oviedo, 33004 Oviedo, Spain; castromaria@uniovi.es (M.P.C.-G.); \\ rey@uniovi.es (M.Á.R.-R.) \\ 2 Department of Exploitation and Prospecting Mines, University of Oviedo, 33004 Oviedo, Spain; \\ tjalonso@uniovi.es \\ * Correspondence: marcosgerman@uniovi.es; Tel.: +34-985-10-82-07 \\ + Presented at the 2nd International Research Conference on Sustainable Energy, Engineering, Materials and \\ Environment (IRCSEEME), Mieres, Spain, 25-27 July 2018.
}

Published: 6 November 2018

\begin{abstract}
In this article the methodology for the determination of the theoretical gamma spectrum originated by applying DGNAA (delayed gamma neutron analysis activation) techniques for the elemental analysis of ash from thermal power plants is exposed. This is a study must be prior to the experimentation in order to define the parameters of the test to obtain the optimal conditions for the detection of each element present in the sample.
\end{abstract}

Keywords: ash; power plant; neutron activation analysis; neutron; DGNAA

\section{Introduction}

In this article the methodology for the determination of the theoretical gamma spectrum originated by applying DGNAA (delayed gamma neutron analysis activation) techniques for the elemental analysis of ash from thermal power plants is exposed. This gamma spectrum depends on the constitution of the sample, as well as on the parameters of the analysis. The objective of the study is to offer a procedure to determine what the parameters of the analysis will be so that the result obtained is the quantitative determination of the different impurities present in the ashes.

The neutron activation is based on the study of the gamma radiation that occurs with simultaneity (Prompt Gamma Neutron Activation Analysis, PGNAA) or later, (Delayed Gamma-Ray Neutron Activation Analysis, DGNAA) to the irradiation of the sample with neutrons When neutrons bombard the sample, they interact with the isotopes present and, generate a reaction that gives rise to an isotope, which can be stable or unstable. If it is unstable, it will return to its stable state in a process called decay, which has a defined duration characterized by half live T1/2. The decay type is characteristic of the isotope and, in some cases, generates a gamma radiation that allows the element to be identified due to the emission energy value gamma and at the time of its decay, it also allows quantifying its presence due to the intensity of the emission.

\section{Materials and Methods}

The National Nuclear Data Center, belonging to the scientific office of the Department of Energy of the United States of America, collects on its website (www.nndc.bnl.gov), among others, the database called "Experimental Nuclear Reaction Data" EXFOR/CSISRS, which contains a very 
extensive collection of experimental data relating to more than 15,500 nuclear reactions. The reactions produced depend on the flow and energy of the neutrons emitted by the source. From the study of the reactions that take place between the sample and the neutrons, the characteristics of the radiations emitted after the bombardment (delayed gamma) can be determined [1]. This determination is made through another database, the NUDAT that offers detailed information on the decay characteristics of the reaction products. It is also possible to access these data through the International Atomic Energy Agency IAEA [2,3].

The reaction of the neutron with the isotope is of a statistical nature, there is a probability (called the cross section $\sigma$ ) of its happening, and this probability is linked to the neutron energy. The cross section of the interaction between the elements and the neutrons depends on the energy of the incident neutrons. The energy of the neutrons depends on the type of source and the materials interposed between the sample and the neutron source.

It should be based on the typical composition of the elements present in the sample. In this case, we start with a composition measured as indicated in the Table 1.

Table 1. Chemical composition of the standard ash.

\begin{tabular}{cc}
\hline Oxides & Contens \\
\hline $\mathrm{SiO}_{2}$ & 66.86 \\
$\mathrm{Al}_{2} \mathrm{O}_{3}$ & 18.08 \\
$\mathrm{Fe}_{2} \mathrm{O}_{3}$ & 5.39 \\
$\mathrm{CaO}$ & 2.45 \\
$\mathrm{MgO}$ & 0.89 \\
$\mathrm{Na} 2$ & 0.79 \\
$\mathrm{~K} 2 \mathrm{O}$ & 1.69 \\
$\mathrm{Mn}_{2} \mathrm{O}_{5}$ & 0.07 \\
$\mathrm{TiO}_{2}$ & 0.88 \\
$\mathrm{P}_{2} \mathrm{O}_{5}$ & 0.15 \\
$\mathrm{BaO}_{2}$ & 0.09 \\
$\mathrm{SrO}_{2}$ & 0.04 \\
\hline
\end{tabular}

To irradiate a sample, it is necessary to use equipment that allows it to be placed as close as possible to the neutron source. Once irradiated the sample should be placed in front of gamma ray detector.

If any of the isotopes present in the sample reacts with the neutrons giving rise to unstable isotopes, the number of atoms created is characterized by the following formula $[4,5]$.

$$
A_{0}=N_{0} \cdot \emptyset \cdot \sigma \cdot\left(1-e^{-\lambda \cdot t_{i}}\right)
$$

where:

- $A_{0}$ number of unstable isotopes created.

- No atoms of each isotope present in the sample that are going to be bombarded by the neutrons of the source. Depends on the concentration of the element in the sample and the isotopic composition.

- $\emptyset$ neutron flux from the source. It is considered constant for all trials.

- $\sigma$ cross section of each reaction produced that results in an unstable isotope.

- $\lambda$ decay constant.

- $t_{i}$ irradiation time.

After irradiating the samples with neutrons, a time elapses, called the delay time $t$. $d$ before starting the measurement, during which the number of unstable atoms is decreasing. At the beginning of the measurement, the number of unstable atoms is given by the following equation:

$$
A_{1}=N_{0} \cdot \emptyset \cdot \sigma \cdot\left(1-e^{-\lambda \cdot t_{i}}\right) \cdot e^{-\lambda \cdot t_{d}}
$$


After a measurement time, the number of unstable atoms will be

$$
A_{2}=N_{0} \cdot \emptyset \cdot \sigma \cdot\left(1-e^{-\lambda \cdot t_{i}}\right) \cdot e^{-\lambda \cdot\left(t_{d}+t_{m}\right)}
$$

where:

- $\quad t_{d}$ delay time.

- $t_{m}$ measurement time.

- $A_{1}$ number of unstable isotopes.

- $A_{2}$ number of unstable isotopes.

Once both the irradiation time and the delay time of the measurement have been set, the isotopic activity can be represented in a system of Cartesian axes against energy, thus obtaining a theoretical spectrum of said concentration. The Figure 1 shows the temporal evolution of the number of unstable atoms.

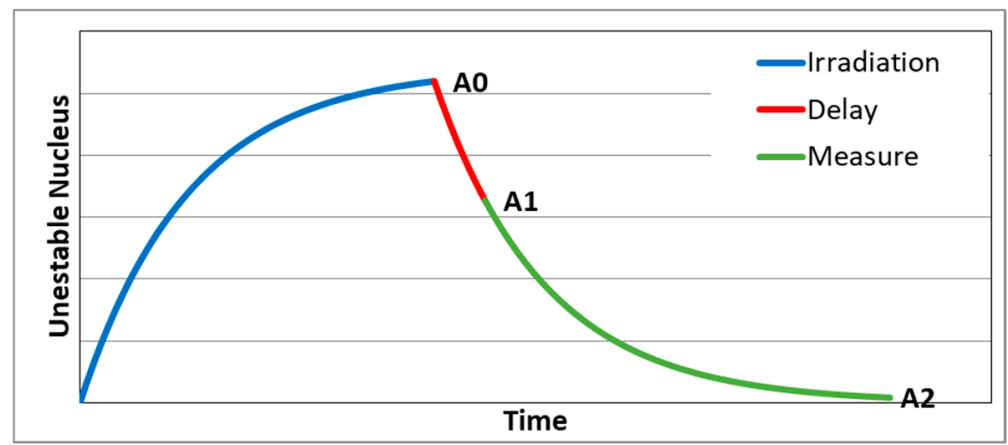

Figure 1. Evolution of number of unstable atoms over time.

\section{Result}

As a result of the study a specific database for the ash sample has been made, and the theoretical spectrum has been calculated for different conditions of the experiment, that is, varying the irradiation time, delay and measurement to achieve a better definition of the spectrum lines that represent the elements we want to identify.

For short irradiation times, the theoretical spectrum is shown in Figure 2.

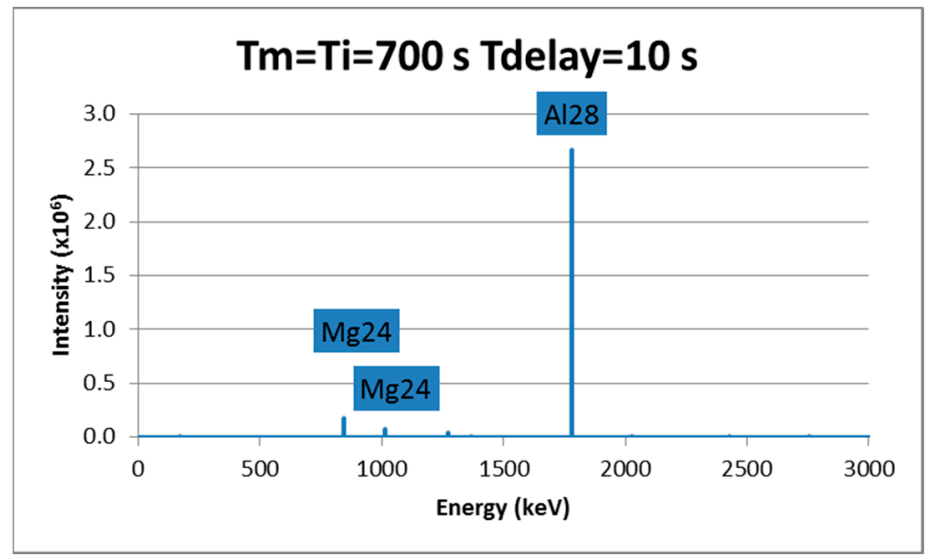

Figure 2. Theoretical spectrum for $t m=t i=700 \mathrm{~s}$ and $t d=10 \mathrm{~s}$ standard ash.

For short irradiation times, the theoretical spectrum is shown in the Figure 3.

In order to study the intensities obtained, we must consider all the reactions that give rise to an unstable isotope. For example the Al-28 is created bombing Al-27, P-31 or Si-28 with neutrons. The reactions have different cross sections, being much higher for the Si-28. The signal from the Si-28 is 
2057 times higher than that from the P-31 and 2820 times the signal from the Al-27 for the chemical concentrations exposed.

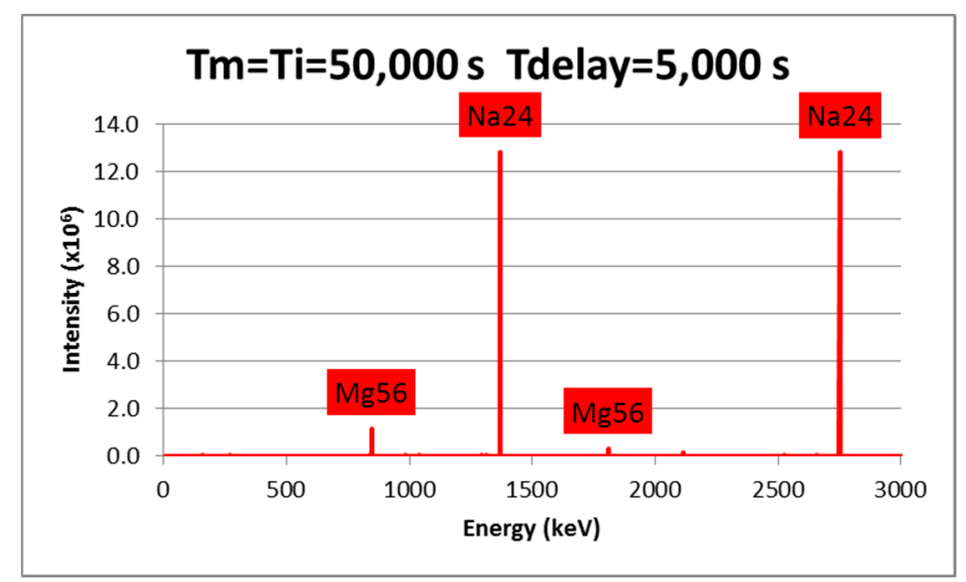

Figure 3. Theoretical spectrum for $t m=t i=50,000 \mathrm{~s}$ and $t d=5000 \mathrm{~s}$ standard ash.

\section{Conclusions}

A methodology has been carried out to obtain a theoretical spectrum of gamma emission from samples bombarded with neutrons

The circumstances that allow us to visualize the desired elements in the best possible way have been analyzed.

For long delay times, the detector can be placed further away from the radioactive source, minimizing background noise.

The use of long delay times eliminates elements with small half-lives from the spectrum. And short measurement times with short delay times eliminates elements with high half-lives.

The efficiency of the gamma radiation detector and the difficulty of capturing all the gamma rays not been taken into account.

Acknowledgments: This study is funded by "Programa Estatal de Investigación, Desarrollo e Innovación orientada a los Retos de la Sociedad. Convocatoria 2016". Ministerio de Economía y Competitividad. Reference: CTQ2016-77887-C2-1-R.

\section{References}

1. EXFOR: Experimental Nuclear Reaction Data. Available online: https://www-nds.iaea.org/exfor/exfor.htm (accessed on 10 July 2018).

2. Livechart-Table of Nuclides-Nuclear Structure and Decay Data. Available online: https://wwwnds.iaea.org/relnsd/vcharthtml/VChartHTML.html (accessed on 10 July 2018).

3. Nudat 2. Available online: http://www.nndc.bnl.gov/nudat2/ (accessed on 10 July 2018).

4. Rey-Ronco, M.A.; Alonso-Sánchez, T.; Castro-García, M.P. Production of $16 \mathrm{~N}$ and obtaining of its gamma spectrum in order to calibrate detectors or determination of fluorine in geological specimens. Nucl. Instrum. Methods Phys. Res. Sect. B Beam Interact. Mater. At. 2010, 268, 2766-2772.

5. Rey-Ronco, M.A.; Alonso-Sánchez, T.; Castro-García, M.P. Mathematical study to improve the sensitivity in the neutron activation analysis of fluorspar. J. Math. Chem. 2010, 48, 165-174. 